\title{
Energy dependence of normal branch oscillations in Scorpius X-1
}

\author{
J. Wang ${ }^{1}$, H.-K. Chang ${ }^{1,2}$, and C.-Y. Liu ${ }^{2,3}$ \\ ${ }^{1}$ Institute of Astronomy, National Tsing Hua University, 30013 Hsinchu, Taiwan \\ e-mail: jwang@mx.nthu.edu.tw \\ 2 Department of Physics, National Tsing Hua University, 30013 Hsinchu, Taiwan \\ 3 LESIA, Paris Observatory, 92195 Meudon, France
}

Received 17 July 2012 / Accepted 5 October 2012

\section{ABSTRACT}

\begin{abstract}
We report the energy dependence of normal branch oscillations (NBOs) in Scorpius X-1, a low-mass X-ray binary Z-source. Three characteristic quantities (centroid frequency, quality factor, and fractional root-mean-squared (rms) amplitude) of a quasi-periodic oscillation signal as functions of photon energy are investigated. We found that, although it is not yet statistically well established, there is a signature indicating that the NBO centroid frequency decreases with increasing photon energy when it is below 6-8 keV, which turns out to be positively correlated with the photon energy at the higher energy side. In addition, the rms amplitude increases significantly with the photon energy below $13 \mathrm{keV}$ and then decreases in the energy band of $13-20 \mathrm{keV}$. There is no clear dependence on photon energy for the quality factor. Based on these results, we suggest that the NBO originates mainly in the transition layer.
\end{abstract}

Key words. accretion, accretion disks - stars: individual: Scorpius X-1 - stars: neutron - X-rays: stars

\section{Introduction}

The bright persistent neutron star low-mass X-ray binary (LMXB) Scorpius X-1 traces a "Z" track in the X-ray color-color diagram (CCD), which consists of three branches - the horizontal branch (HB), the normal branch (NB), and the flaring branch (FB, Hasinger \& van der Klis 1989). The typical Fourier power spectra in Scorpius X-1 are composed of distinct aperiodic variabilities in most of these branches, including noise components (broad structure) and quasi-periodic oscillations (QPOs, narrow feature). There are three distinct types of QPOs observed by the Rossi X-Ray Timing Explorer (RXTE), i.e., the normal branch oscillation (NBO) at $\sim 6 \mathrm{~Hz}$, the horizontal branch oscillation (HBO) at $\sim 45 \mathrm{~Hz}$ with a harmonic at about $90 \mathrm{~Hz}$, and the kiloherz (kHz) QPOs (van der Klis et al. 1996, 1997).

The NBOs in Scorpius X-1, which occur on the mid- and lower NB, show some correlations with the variability of the $\sim 45 \mathrm{~Hz} \mathrm{HBO}$ and the twin kHz QPOs (van der Klis et al. 1996; Yu 2007), and therefore imply some kind of coupling between the three types of QPOs (van der Klis et al. 1996; Dieters \& van der Klis 2000). The NBO frequency remains approximately constant at about $6 \mathrm{~Hz}$, in general. However, the timing properties along the $\mathrm{Z}$ track show that the NBO frequency extends to at most $\sim 21 \mathrm{~Hz}$ and its position moves smoothly into the lower part of the FB (Priedhorsky et al. 1986; Dieters \& van der Klis 2000). This smooth transition indicates that the NBO and flaring branch oscillation (FBO) in Scorpius X-1 are physically related to each other (Kuulkers \& van der Klis 1995; Dieters \& van der Klis 2000; Casella et al. 2006). Properties of both the twin $\mathrm{kHz}$ QPO and $45 \mathrm{~Hz} \mathrm{HBO}$ depend on the NBO flux. The upper $\mathrm{kHz}$ QPO frequency is anticorrelated with the NBO flux, and the lower $\mathrm{kHz}$ QPO becomes stronger when the NBO flux is low (Yu et al. 2001). Significant HBOs are detected during the NBO phase of high flux, while the HBO disappears at the low flux of NBO phase (Yu 2007). The coupling between the properties of $\mathrm{kHz}$ QPO and HBOs and the phase of the NBO makes the NBO a unique phenomenon.
The photons from different regions of the accretion disk carry different energies and present distinct physical properties. So the QPOs detected in different energy band may have varying characteristics. Inspired by the idea that the photon energy dependence of QPOs may provide some additional information on the nature of QPOs, and limited by the available data that provide sufficient spectral information and at the same time include QPO signals, we performed a systematical investigation of the energy dependence of NBOs. We describe our data reduction and analysis in Sect. 2. Results are presented in Sect. 3. In Sect. 4, we discuss the physical implications of our result. Section 5 contains a short summary.

\section{Data reduction and analysis}

To investigate the energy dependence of QPOs in Scorpius X-1, high time resolution data with sufficient spectral information are needed. Quasi-periodic oscillations signals should also be present in these data. We searched all RXTE observations of Scorpius X-1 for suitable data. Among these, two binned data modes, i.e., B_4ms_16A_0_249_H and B_4ms_16B_0_249_H, which have a 4-ms time resolution and 16 energy bands consisting of all PCA channels (from original channel 0 to 249) binned with two different schemes (A and B), provide adequate timing and spectral information for our purpose. We examined all data recorded in these two modes and selected those with QPO signals present (with quality factor $Q$ higher than 2) in the energy band of channel $0-63$, which roughly corresponds to 2 to $20 \mathrm{keV}$. The selected data are listed in Table 1 . We further more defined six energy bands, as shown in Table 2, for the energy dependence study. An HBO signal was found in the highest energy band defined in Table 2 in the data set 30035-01-07-00, but it cannot be detected in the original 0-63 channel. In addition, another $\sim 45 \mathrm{~Hz} \mathrm{HBO}$ signal was detected in the original 0-63 channel in 93082-01-02-05, but it cannot be found in any individual energy band defined in Table 2. We also found 
Table 1. Data log.

\begin{tabular}{lcccccc}
\hline \hline ObsID & Start & End & $\begin{array}{c}\text { Exposure } \\
(\mathrm{s})\end{array}$ & PCU & $\begin{array}{c}\text { Rate } \\
\text { (cts/s/PCU) }\end{array}$ & Seg \\
\hline 10056-01-04-00 & $96-05-27$ 20:42:05 & $27 / 05 / 9623: 02: 13$ & 2633 & 5 & 18776 & All \\
10056-01-04-02 & $96-05-2804: 26: 25$ & $28 / 05 / 9610: 29: 13$ & 10500 & 5 & 17685 & All \\
10056-01-05-00 & $96-05-2811: 11: 04$ & $28 / 05 / 9614: 23: 13$ & 5277 & 4 & 16469 & All \\
& & & 375 & 5 & & \\
30035-01-02-000 & $98-05-3100: 26: 47$ & $31 / 05 / 9808: 26: 47$ & 77 & 3 & 18182 & 4,9 \\
& & & 2936 & 5 & & \\
30035-01-05-00 & $98-06-0100: 26: 55$ & $01 / 06 / 9801: 18: 14$ & 656 & 3 & 18413 & All \\
30035-01-07-00 & $98-07-0206: 26: 32$ & $02 / 07 / 9810: 27: 14$ & 4968 & 5 & 16486 & 1,2 \\
$93082-01-01-01$ & $09-06-1623: 43: 14$ & $17 / 06 / 0900: 56: 18$ & 48 & 4 & 14718 & All \\
& & & 926 & 5 & & \\
$93082-01-01-03$ & $09-06-1801: 40: 22$ & $18 / 06 / 0902: 21: 18$ & 938 & 5 & 14348 & All \\
$93082-01-01-04$ & $09-06-1705: 13: 12$ & $17 / 06 / 0906: 01: 18$ & 1556 & 5 & 14486 & All \\
$93082-01-02-05$ & $09-06-2304: 07: 09$ & $23 / 06 / 0904: 40: 18$ & 1311 & 3 & 13447 & All \\
\hline
\end{tabular}

Notes. Here listed are the RXTE observation identification number (ObsID), their start and stop time, the exposure time in seconds of the light curve after screening (on elevation and time_since_SAA) and selection of NBO signal presence, the number of working PCU, the original 0-63 channels count rate in units of cts/s/PCU and the segments with NBO signals.

Table 2. RXTE/PCA energy bands used in this work.

\begin{tabular}{lcc}
\hline \hline \multicolumn{3}{c}{ The observations in 1996 and 1998 } \\
\hline $\begin{array}{l}\text { PCA } \\
\text { channel }\end{array}$ & $\begin{array}{c}\text { Energy band } \\
(\mathrm{keV})\end{array}$ & $\begin{array}{c}\text { Centroid energy } \\
(\mathrm{keV})\end{array}$ \\
\hline $0-13$ & $1.83-5.07$ & 2.54 \\
$14-19$ & $5.07-7.24$ & 6.16 \\
$20-22$ & $7.24-8.34$ & 7.79 \\
$23-28$ & $8.34-10.52$ & 9.43 \\
$29-35$ & $10.52-13.09$ & 11.81 \\
$36-63$ & $13.09-23.46$ & 18.26 \\
\hline \multicolumn{3}{c}{ The observations in 2009 } \\
\hline PCA & Energy band & Centroid energy \\
channel & $(\mathrm{keV})$ & $(\mathrm{keV})$ \\
\hline $0-11$ & $2.18-5.26$ & 2.63 \\
$12-15$ & $5.26-7.02$ & 6.14 \\
$16-19$ & $7.02-8.78$ & 7.90 \\
$20-22$ & $8.78-10.10$ & 9.44 \\
$23-30$ & $10.10-13.60$ & 11.85 \\
$31-63$ & $13.60-28.40$ & 21.00 \\
\hline
\end{tabular}

another data set, 30035-01-01-00, which shows the $\sim 45 \mathrm{~Hz}$ HBO signal in the original 0-63 channels, but has no NBO. In a more detailed examination of this data set, its HBO signal was only detected in the original $0-16$ channels but not in higher energy bands. We therefore did not include this data set in this work. Limited by the available data, we studied only NBOs. Some of those data sets include several segments. We only exploited the segments with an NBO signal (see Table 1 for details).

The data were reduced with the HEASOFT package version 6.8. Only data with the elevation angle $>10^{\circ}$ and the time since South Atlantic Anomaly (SAA) $<0$ or $>30$ min were selected for further analysis. We extracted light curves with a time resolution of $4 \mathrm{~ms}\left(2^{-8} \mathrm{~s}\right)$ in the six energy bands defined in Table 2. Using the Leahy normalization (Leahy et al. 1983), we computed the power density spectra (PDS) without subtracting white noise. The average power expected from a Poisson distribution is 2 . The original $4 \mathrm{~ms}\left(2^{-8} \mathrm{~s}\right)$ time resolution was used, and each continuous set was divided into segments of 8192 bins. A fast Fourier transform (FFT) algorithm for each segment was computed, yielding a frequency PDS in the range of $3 \times 10^{-2}-128 \mathrm{~Hz}$. We calculated the average PDS for

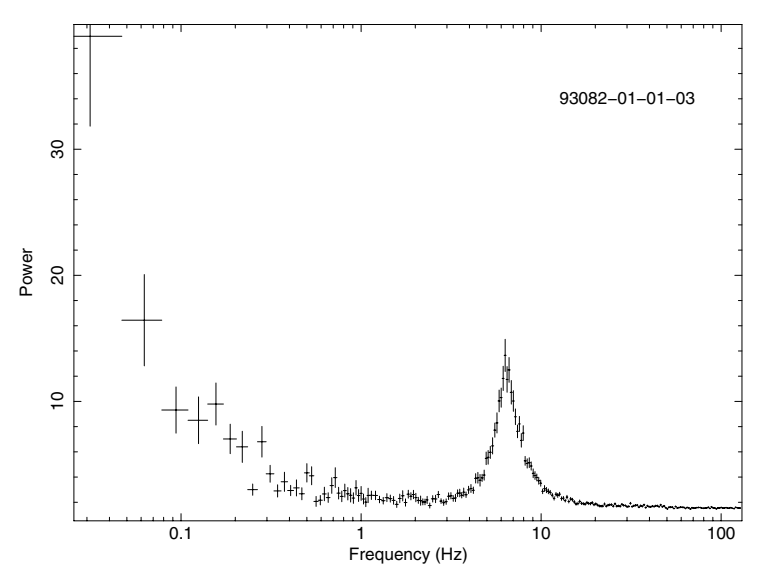

Fig. 1. One example of PDS which shows a $\sim 6 \mathrm{~Hz}$ NBO. The PDS is produced in the PCA original channels $0-63$. The mean count rate is $14348 \mathrm{cts} / \mathrm{s} / \mathrm{PCU}$. The exposure time is $938 \mathrm{~s}$.

each continuous data interval. A logarithmic rebinning of -1.02 was used for the rebin option. Figure 1 shows an example of the $\sim 6 \mathrm{~Hz}$ NBO signal.

We employed three components to fit the resulting PDS: a Lorenzian for the very low frequency noise (VLFN), a power law for the white noise, and another Lorentzian for the NBO. All fits were performed using the $\chi^{2}$ minimization technique. The errors of the analytic model parameters were obtained at the $\chi^{2}$ variation of 2.70 , which corresponds to a $90 \%$ confidence for a single parameter.

A QPO signal is characterized by three quantities: the centroid frequency (representing the position of a QPO), the quality factor (characterizing the coherence of a QPO signal), and the fractional root-mean-squared (rms) amplitude (a measure of the signal strength, which is proportional to the square root of the peak power contribution to the PDS). From the fitting we obtained three parameters of NBOs, i.e., the oscillation amplitude, the centroid frequency, and the full width at half maximum (FWHM). The quality factor was then computed according to its definition (centroid frequency/FWHM). The rms amplitude was calculated by the integral of the normalized Lorenzian PDS in the appropriate frequency range and taking the mean count rate of the source into account. Details of the rms determination can be found in Lewin et al. (1988) and van der Klis (1989). The 
Table 3. Fitting results of a two-power-law description $\left(v(E)=a E^{\Gamma_{1}}+b E^{\Gamma_{2}}\right)$ for the energy dependence of the centroid frequency of NBOs in Scorpius X-1.

\begin{tabular}{|c|c|c|c|c|c|c|c|}
\hline ObsID & $\Gamma_{1}$ & $a$ & $\Gamma_{2}$ & $b$ & $\chi_{v}^{2}$ & $\chi_{v, C}^{2}$ & $\chi_{v, Q}^{2}$ \\
\hline 10056-01-04-00 & $-0.17 \pm 0.058$ & $3.40 \pm 0.65$ & $0.11 \pm 0.028$ & $3.05 \pm 0.39$ & 0.19 & 0.26 & 0.55 \\
\hline 10056-01-04-02 & $-0.22 \pm 0.026$ & $3.65 \pm 0.29$ & $0.15 \pm 0.030$ & $2.79 \pm 0.43$ & 0.13 & 0.58 & 0.15 \\
\hline 10056-01-05-00 & $-0.62 \pm 0.023$ & $2.52 \pm 0.30$ & $0.086 \pm 0.025$ & $5.03 \pm 0.38$ & 1.03 & 2.58 & 0.26 \\
\hline 30035-01-02-000 & $-0.42 \pm 0.038$ & $2.32 \pm 0.430 .083 \pm 0.022$ & $4.38 \pm 0.29$ & 0.02 & 0.51 & 0.27 & \\
\hline 30035-01-05-00 & $-0.43 \pm 0.11$ & $0.81 \pm 1.13$ & $0.040 \pm 0.054$ & $5.23 \pm 0.77$ & 0.65 & 0.70 & 0.37 \\
\hline $30035-01-07-00$ & $-3.58 \pm 0.27$ & $4.27 \pm 0.30$ & $0.016 \pm 0.014$ & $6.22 \pm 0.20$ & 0.37 & 0.86 & 0.11 \\
\hline 93082-01-01-01 & $-0.24 \pm 0.052$ & $5.87 \pm 0.70$ & $0.35 \pm 0.050$ & $1.38 \pm 0.68$ & 0.36 & 1.17 & 0.35 \\
\hline 93082-01-01-03 & $-0.16 \pm 0.084$ & $3.16 \pm 1.00$ & $0.082 \pm 0.029$ & $3.65 \pm 0.46$ & 0.15 & 0.18 & 0.49 \\
\hline 93082-01-01-04 & $-0.41 \pm 0.057$ & $3.52 \pm 0.85$ & $0.10 \pm 0.042$ & $4.19 \pm 0.68$ & 0.62 & 1.16 & 0.65 \\
\hline 93082-01-02-05 & $-0.29 \pm 0.067$ & $3.11 \pm 0.78$ & $0.11 \pm 0.025$ & $3.56 \pm 0.35$ & 0.29 & 0.57 & 0.50 \\
\hline
\end{tabular}

Notes. In Col. $7\left(\chi_{v, C}^{2}\right)$, listed is the reduced $\chi^{2}$ of fitting with a constant centroid frequency. In Col. $8\left(\chi_{v, Q}^{2}\right)$, the reduced $\chi^{2}$ of fitting the quality factor (Fig. 3) with a constant is shown.
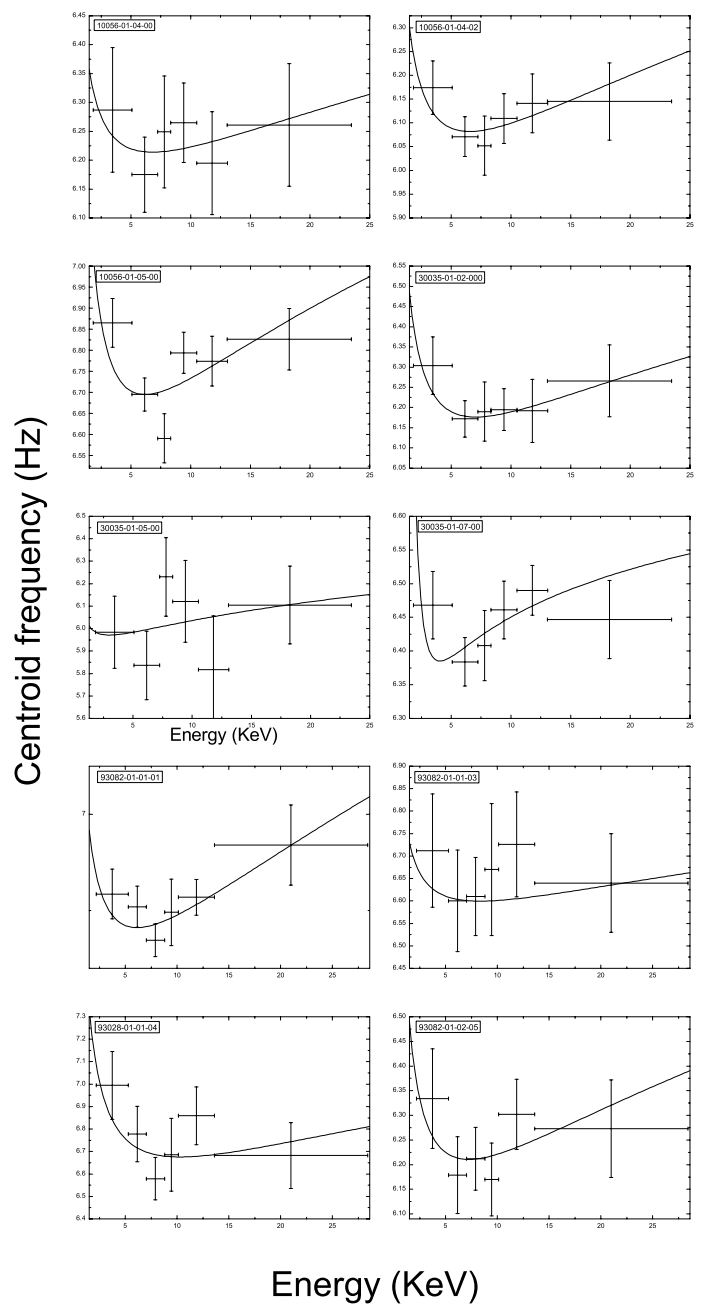

Fig. 2. Centroid frequency versus photon energy for NBOs in Scorpius X-1. The solid lines are the best fits with a two-power-law function to the data.

errors of the quality factor and rms amplitude were estimated with a standard error propagation (Bevington \& Robinson 2003).

\section{Results}

We investigated the photon energy dependence of three characteristic quantities (i.e., the centroid frequency, quality factor, and rms amplitude) of NBOs. Figure 2 shows the energy dependence of the centroid frequency. Although error bars are large, there seems to be a common trait in almost all data sets that the dependence evolves from a negative correlation to a positive one with increasing photon energy. We therefore adopted a two-powerlaw function, $v(E)=a E^{\Gamma_{1}}+b E^{\Gamma_{2}}$, to describe the relation between the centroid frequency $v$ (in units of $\mathrm{Hz}$ ) and the photon energy $E$ (in units of $\mathrm{keV}$ ). The fitting results are listed in Table 3. In all data set we have a negative and a positive power index, which together cause the centroid frequency to decrease with increasing energy first, and beyond about $6-8 \mathrm{keV}$ it turns to be positively correlated with the photon energy. This dependence is not obvious in one data set (ObsID 30035-01-05-00) among the ten under study. While the low $\chi_{v}^{2}$ value indicates that this description is only suggestive because of the large error bars, the appearance of this trait in almost all data sets asserts its reality. Fitting this energy dependence with a constant frequency also gives acceptable results, except for the data set of ObsID 10056-01-05-00. The reduced $\chi^{2}$ of this constantfrequency fitting is listed in Col. 7, denoted with $\chi_{v, C}^{2}$, in Table 3. However, with the poor fitting result of this data set to a constant frequency and with the appearance of this "V" shape trait in almost all data sets, the signature indicating a "V" shape dependence of the centroid frequency on the photon energy should not be overlooked. Future observations with better statistics to confirm or disprove this phenomenon are highly necessary.

Figure 3 shows the energy dependence of the quality factor. We do not see any clear relation between the quality factor and the photon energy. The reduced $\chi^{2}$ of a constant-quality-factor fitting is listed in Col. 8, denoted with $\chi_{v, Q}^{2}$, in Table 3. Based on these fitting results, it is not inconsistent with a constant quality factor. We note, however, that because of the large error bars, all fittings give very low reduced $\chi^{2}$ values. This means that these data sets are not sufficiently constrained.

In Fig. 4 we can see that the rms amplitude increases monotonically with the photon energy except for the highest energy band. This means that the strength of the NBO signal increases with the photon energy. The rms amplitude increases significantly with the photon energy below $13 \mathrm{keV}$. However, the increase of rms amplitude with photon energy seems to stop when the photon energy is higher than $\sim 13 \mathrm{keV}$, and there might be a plateau. We do not have enough photons at even higher energies for our study. It would be interesting to know beyond which energy the rms amplitude starts to decrease. To describe the increase of the rms amplitude with photon energy, we used a power law relation, $\operatorname{rms}(E)=C E^{\Gamma}$, to fit the data points in the lower five energy bands. Table 4 shows the fitting results. 

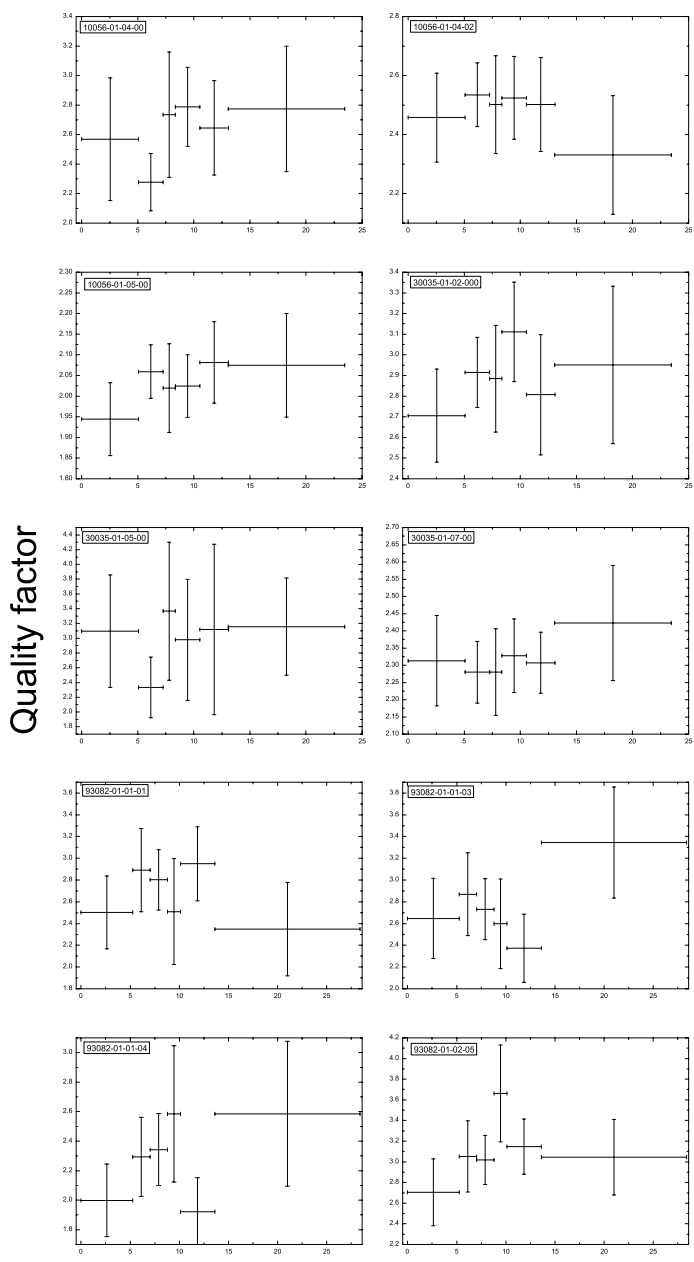

Energy (KeV)

Fig. 3. Quality factor versus photon energy for NBOs in Scorpius X-1.

Table 4. Fitting results of a power law $\left(\operatorname{rms}(E)=C E^{\Gamma}\right)$ for the increase of rms amplitude of NBOs in Scorpius X-1.

\begin{tabular}{lccc}
\hline \hline ObsID & $\Gamma$ & $C$ & $\chi_{v}^{2}$ \\
\hline 10056-01-04-00 & $0.78 \pm 0.18$ & $1.06 \pm 0.40$ & 0.27 \\
10056-01-04-02 & $0.71 \pm 0.09$ & $1.92 \pm 0.34$ & 0.44 \\
10056-01-05-00 & $0.75 \pm 0.06$ & $1.77 \pm 0.22$ & 0.76 \\
30035-01-02-000 & $0.73 \pm 0.12$ & $1.27 \pm 0.30$ & 0.30 \\
30035-01-05-00 & $0.63 \pm 0.34$ & $1.12 \pm 0.80$ & 0.14 \\
30035-01-07-00 & $0.70 \pm 0.07$ & $1.79 \pm 0.25$ & 3.13 \\
93082-01-01-01 & $0.70 \pm 0.19$ & $1.52 \pm 0.61$ & 0.36 \\
93082-01-01-03 & $0.74 \pm 0.20$ & $1.52 \pm 0.66$ & 0.28 \\
93082-01-01-04 & $0.72 \pm 0.18$ & $1.44 \pm 0.56$ & 0.18 \\
93082-01-02-05 & $0.70 \pm 0.16$ & $1.65 \pm 0.55$ & 0.24 \\
\hline
\end{tabular}

Notes. Only the five lower energy bands are included in the fitting.

Roughly speaking, the rms amplitude increases with photon energy according to a power law of power index about 0.7 before reaching a possible plateau. In the data set 30035-01-07-00 the plateau seems to start at a lower energy.

\section{Discussions}

The mechanism of NBOs was suggested to be related to the radial oscillation during radiation feedback (Fortner et al. 1989; Miller \& Park 1995) or the different modes of disk oscillations
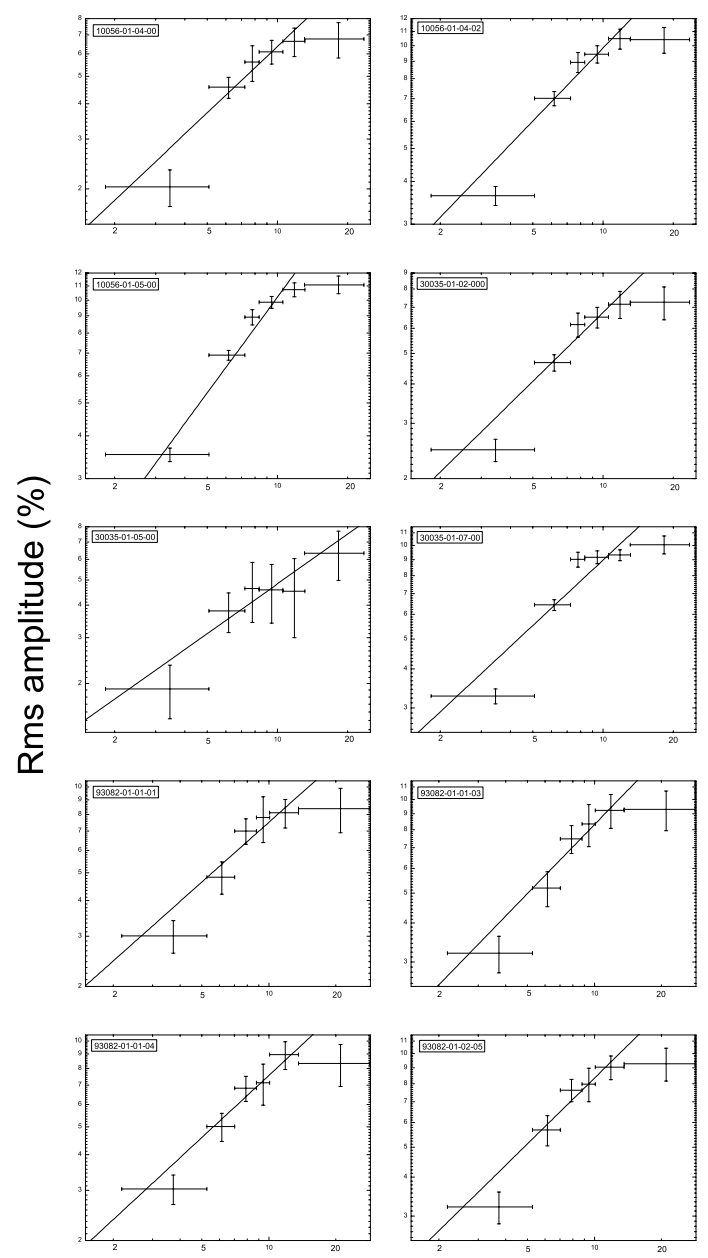

Energy (KeV)

Fig. 4. Rms amplitude versus photon energy for NBOs in Scorpius X-1. The solid lines are the best fits with a power law to the data points in the five lower energy bands.

(Alpar et al. 1992; Titarchuk et al. 2001; Bildsten \& Cutler 1995; Bildsten et al. 1996; Horák et al. 2004). The standard explanation attributes the $\sim 6 \mathrm{~Hz} \mathrm{NBO}$ to part of the accretion with a near Eddington accretion rate, in an approximately spherically symmetric radial inflow at a radius of $\sim 100 \mathrm{~km}$ (van der Klis 1995; Hasinger et al. 1990). According to the radiation hydrodynamic model (Fortner et al. 1989), NBO originates from a radiationforce/opacity feedback loop within a spherical flow region at about $300 \mathrm{~km}$ from the neutron star surface. The frequency of the oscillations depends on the luminosity. Alternatives are the acoustic oscillation in a thick disk due to the density and opticalthickness perturbations caused by the rotating medium in a subsonic region of the accretion disk (the frequency is determined by the rotation rate and vorticity, Alpar et al. 1992), g-mode oscillations arising from the thermal buoyancy in the ocean on rotating neutron stars (Bildsten \& Cutler 1995; Bildsten et al. 1996), the low-frequency modulation in a nonlinear resonance oscillation of the relativistic disk (Horák et al. 2004), and the acoustic oscillations of a spherical shell surface within $\sim 20 \mathrm{~km}$ of the neutron star surface because of the change of accretion geometry in the transition zone at high accretion rate (Titarchuk et al. 2001). All of these interpretations focus on the $\sim 6 \mathrm{~Hz}$ frequency and its dependence on the accretion rate. None of them can explain the energy dependence of the centroid frequency (if it is further confirmed) and of the rms amplitude. 
In the NBOs of Scorpius X-1, the rms amplitude has a strong energy dependence and the centroid frequency is also likely to have a "V" shape energy dependence. The centroid frequency changes from anticorrelation to a positive correlation at about 6-8 keV. This nonmonotonic energy dependence of the centroid frequency implies that the emission zone of the NBOs experiences a radial variation during the accretion process. The rms amplitude increases monotonically and significantly with the photon energy below $\sim 13 \mathrm{keV}$. When the photon energy is higher than $13 \mathrm{keV}$, the rms amplitude seems to stop increasing, which may indicate that the NBO signals reach the strongest strength at 13-20 keV. Higher energy photons are often emitted from the inner region of an accretion disk. Consequently, the most likely region responsible for such physics can be referred to the transition zone between the inner boundary of accretion disk and the magnetosphere, in which the transition of the properties for accretion flow occurs (Titarchuk et al. 1998; Titarchuk \& Osherovich 1999; Titarchuk et al. 1999). Normal branch oscillation occurs at high accretion rates, i.e., near the Eddington rate. The high-mass flux deposits increasingly more matter in the transition zone, leading to the expansion and the radial scale change of this region. However, the pile of more deposits with greater viscosity can contribute to the radiation enhancement, as well as to the emission of photons with higher energies. As a result, the radial scale change of the transition zone may be responsible for the observed nonmonotonic energy dependence of the centroid frequency. In addition, because of the limited region of the transition zone, more deposits enhance the viscosity and thus suppress the further monotonically increasing strength of the NBO signal, which can explain the energy dependence of rms amplitude at high energy. We therefore suggest that the NBOs are a type of oscillations in the transition zone. This kind of oscillation may originate in the transition layer due to the viscosity of clumps (e.g. Titarchuk et al. 2001). On the other hand, this oscillation may also carry the information of frequency oscillation modes in the accretion disk and manifest itself as modulation of the accretion rate during the course when disk matter penetrates the transition layer (Paczynski 1987; Nowak \& Wagoner 1993; Abramowicz et al. 2007).

\section{Summary}

We investigated the energy dependence of three characteristic quantities of NBOs in Scorpius X-1. We arrived at the following conclusion:

(1). Although it has yet to be better established statistically, there is a signature indicating that the energy dependence of centroid frequency changes from a negative correlation to a positive one. The turning points are located at $6-8 \mathrm{keV}$.

(2). It seems that the quality factors have no obvious relation with the photon energy, which indicates that the coherence of NBOs is independent of the photon energy.
(3). The rms amplitude increases significantly with the photon energy below $13 \mathrm{keV}$. It seems to reach a plateau beyond that.

(4). The observed energy dependence of three characteristic quantities of NBOs in Scorpius X-1 suggests that the NBOs may be a type of oscillations in the transition layer.

(5). Horizontal branch oscillation signals are present in three data sets (30035-01-07-00, 93082-01-02-05, and 30035-0101-00), but they display a distinct energy dependence. The HBO signal is only detected in the original 36-63 channels for 30035-01-07-00 and in the original 0-16 channels for 30035-01-01-00. For 93082-01-02-05, the HBO is detected in the original 0-63 channels, but not in any individual energy band defined in Table 2. It seems that the energy dependence of HBO signals changes with the state of the source in Scorpius X-1. Additional investigation of this phenomenon will be very rewarding but it requires data of suitable timing and spectral resolution and of sufficiently good statistics.

Acknowledgements. This work was supported by the National Science Council of the Republic China under grant NSC 99-2112-M-007 -017 -MY3.

\section{References}

Abramowicz, M. A., Horák, J., \& Kluźniak, W. 2007, Acta Astron., 57, 1

Alpar, M. A., Hasinger, G., Shaham, J., \& Yancopoulos, S. 1992, A\&A, 257, 627 Bevington, P. R., \& Robinson, D. K. 2003, Data reduction and error analysis for the physical sciences, 3rd edn. (Boston, MA: McGraw-Hill)

Bildsten, L., \& Cutler, C. 1995, ApJ, 449, 800

Bildsten, L., Ushomirsky, G., \& Cutler, C. 1996, ApJ, 460, 827

Casella, P., Belloni, T., \& Stella, L. 2006, A\&A, 446, 579

Dieters, S. W., \& van der Klis, M. 2000, MNRAS, 311, 201

Fortner, B., Lamb, F. K., \& Miller, G. S. 1989, Nature, 342, 775

Hasinger, G., \& van der Klis, M. 1989, A\&A, 225, 79

Hasinger, G., van der Klis, M., Ebisawa, K., Dotani, T., \& Mitsuda, K. 1990, A\&A, 235, 131

Horák, J., Abramowicz, M. A., Karas, V., \& Kluzniak, W. 2004, PASJ, 56, 819

Kuulkers, E., \& van der Klis, M. 1995, A\&A, 303, 801

Leahy, D. A., Darbro, W., Elsner, R. F., et al. 1983, ApJ, 266, 160

Lewin, W. H. G., van Paradijs, J., \& van der Klis, M. 1988, Space Sci. Rev., 46, 273

Miller, G. S., \& Lamb, F. K. 1992, ApJ, 388, 541

Miller, G. S., \& Park, M. G. 1995, ApJ, 440, 771

Nowak, M., \& Wagoner, R. V. 1993, ApJ, 418, 187

Paczyński, B. 1987, Nature, 327, 303

Priedhorsky, W., Hasinger, G., Lewin, W. H. G., et al. 1986, ApJ, 306, L91

Titarchuk, L. G., Bradshaw, C. F., Geldzahler, B. J., \& Fomalont, E. B. 2001 ApJ, 555, L45

Titarchuk, L., \& Osherovich, V. 1999, ApJ, 518, L95

Titarchuk, L., Lapidus, I., \& Muslimov, A. 1998, ApJ, 499, 315

Titarchuk, L., Osherovich, V., \& Kuznetsov, S. 1999, ApJ, 525, L129

van der Klis, M. 1989, in Timing neutron stars, eds. H. Ögelman, \& E. P. J. van den Heuvel, NATO Advanced Science Institutes (ASI) Ser. C, 262, 27

van der Klis, M. 1995, in X-ray Binaries, ed. W. H. G. Lewin, J. van Paradijs, \&

E. P. J. van dn Heuvel (Cambridge: Cambridge Univ. Press), 252

van der Klis, M., Swank, J. H., Zhang, W., et al. 1996, ApJ, 469, L1

van der Klis, M., Wijnands, R. A. D., Horne, K., \& Chen, W. 1997, ApJ, 481, L97

Yu, W. 2007, ApJ, 659, L145

Yu, W., van der Klis, M., \& Jonker, P. G. 2001, ApJ, 559, L29 